\title{
Dynamic Output Feedback Control for Nonlinear Networked Control Systems with Random Packet Dropout and Random Delay
}

\author{
Shuiqing Yu, ${ }^{1,2}$ Junmin Li, $^{1}$ and Yingde Tang ${ }^{1}$ \\ ${ }^{1}$ School of Mathematics and Statistics, Xidian University, Xian 710071, China \\ ${ }^{2}$ School of Statistics, Xi'an University of Finance and Economics, Xian 710100, China \\ Correspondence should be addressed to Shuiqing Yu; yu-shuiqing@126.com
}

Received 6 April 2013; Accepted 9 October 2013

Academic Editor: Piermarco Cannarsa

Copyright (C) 2013 Shuiqing Yu et al. This is an open access article distributed under the Creative Commons Attribution License, which permits unrestricted use, distribution, and reproduction in any medium, provided the original work is properly cited.

This paper investigates the dynamic output feedback control for nonlinear networked control systems with both random packet dropout and random delay. Random packet dropout and random delay are modeled as two independent random variables. An observer-based dynamic output feedback controller is designed based upon the Lyapunov theory. The quantitative relationship of the dropout rate, transition probability matrix, and nonlinear level is derived by solving a set of linear matrix inequalities. Finally, an example is presented to illustrate the effectiveness of the proposed method.

\section{Introduction}

Networked control systems (NCSs) are feedback control loops closed through a real time network. Compared with conventional point-to-point control systems, NCSs have many attractive advantages of low cost, simple installation, reduced wiring, easy maintenance, and so on. On the other hand, the introduction of network also presents some challenging problems such as quantization [1-4], packet dropout [5-8], and time delays [9-11], which bring difficulties for analysis and design of NCSs. Hence, NCSs have attracted considerable research interests in recent years [12-14].

Generally, time delays and packet dropout are two essential issues that need careful consideration in NCSs design. They can be handled separately or simultaneously. Some results have been available. Markov chain and independent Bernoulli random process were commonly used to model the packet loss processes. The results applying the Bernoulli process assume that the packet losses are independent and identically distributed $[5,7]$, while those using the Markov chain assume that the packet losses are burst and occur according to a Markov chain $[6,8]$. Based on a similar Bernoulli random delay model, the controller design problem for NCSs with both sensor-to-controller and controller-toactuator delays has been studied in [9]. A delay-dependent approach for NCSs controller design has been provided in [10], where the feedback gain of a memoryless controller was determined by solving a set of linear matrix inequalities. In $[1,2,11]$, the network-induced random delays were modeled as Markov chains such that the closed-loop system was a jump linear system. In [15], a nonlinear stochastic system model was proposed to describe the NCSs with both random packet dropout and network-induced time-varying delay. Based on this model, a sufficient condition was derived to the exponential stability of the nonlinear NCSs. However, [15] only studied the static output feedback controller and time-varying delay. In this paper, we extend the results in [15] to general random delay and dynamic output feedback controller.

This paper proposes a new nonlinear networked control systems model with both random packet dropout and random delay. In this model, two random variables are included and they are independent of each other. The random delay is modeled as a Markov chain with partly known transition probability matrix, which covers the completely known and completely unknown transition probabilities as its two 
special cases. The random packet dropout is modeled as Bernoulli distributed white sequences. A sufficient condition is presented for the stabilization of the closed systems, and a dynamic output feedback controller is designed based upon the Lyapunov theory. An example is presented to illustrate the effectiveness of the proposed method.

Notation. Throughout the paper, $R^{n}$ denotes the $n$-dimensional Euclidean space. The superscripts " $T$ " and " -1 " stand for the transpose and inverse of a matrix, respectively. $\operatorname{diag}\{\cdots\}$ stands for a block-diagonal matrix. The symbol * denotes the symmetric part in a symmetric matrix. The notation $P>0$ means that $P$ is real symmetric and positive definite matrix. In addition, $E\{x\}$ and $E\{x \mid y\}$ denote expectation of $x$ and $x$ conditional on $y$, respectively. Matrices, if their dimensions are not explicitly stated, are assumed to be compatible for algebraic operations.

\section{Problem Statement and Preliminaries}

The structure of the NCSs to be discussed in this paper is depicted in Figure 1.

The plant model is described by the following nonlinear discrete-time system:

$$
\begin{gathered}
x(k+1)=A x(k)+B u(k)+f(x(k)) \\
y(k)=C x(k),
\end{gathered}
$$

where $x(k) \in R^{n}, u(k) \in R^{m}$, and $y(k) \in R^{p}$ are the state, control input, and measured output, respectively, and $A, B$, $C$ are known real matrices with appropriate dimensions. The function $f(x(k))$ satisfies the following inequality:

$$
f^{T}(x(k)) f(x(k)) \leq \beta^{2} x^{T}(k) G^{T} G x(k),
$$

where $\beta>0$ is a scalar parameter and $G$ is a constant matrix.

Throughout the paper, the following assumptions are needed.

Assumption 1. The state $x(k)$ is not measurable.

Assumption 2. For convenience of analysis, it is assumed that the network induced packet dropout and delay occur only in the sensor-to-controller side.

In what follows, we model signal transmission delay and random packet dropout mathematically.

2.1. Signal Transmission Delay. Let $d_{k}$ represent the random delay from sensor to controller. Assume that $d_{k}$ take the positive integers between $d_{m}$ and $d_{M}$. In real communication systems, current time delay is usually affected by previous time delays. It is reasonable to model the time delay $d_{k}$ as a Markov chain and its transition probability matrix is $\Pi=$ $\left[\lambda_{r s}\right]$; that is, $d_{k}$ jump from mode $r$ to $s$ with probability $\lambda_{r s}$, which is defined by

$$
\lambda_{r s}=P_{r}\left(d_{k+1}=s \mid d_{k}=r\right),
$$

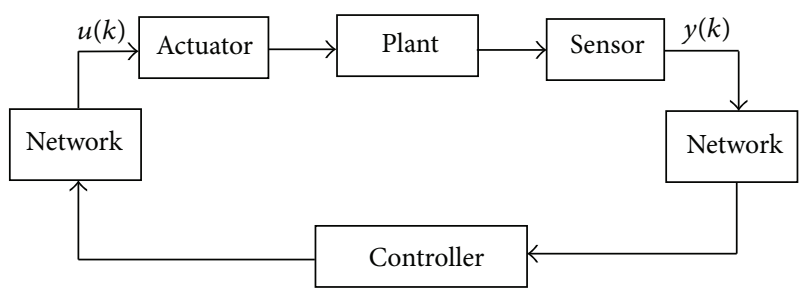

FIGURE 1: Block diagram of the considered NCSs.

where $\lambda_{r s}>0, \sum_{s=d_{m}}^{d_{M}} \lambda_{r s}=1$, for all $r, s \in \Lambda$, where $\Lambda=$ $\left\{d_{m}, d_{m}+1, \ldots, d_{M}\right\}$. In reality, the transition probabilities of the Markov chain in this paper are usually partially available; namely, some elements in matrix $\Pi$ are unknown. For instance, $d_{k}$ with four modes will have the transition probability matrix $\Pi$ as

$$
\Pi=\left[\begin{array}{cccc}
\lambda_{11} & ? & \lambda_{13} & ? \\
? & ? & ? & \lambda_{24} \\
\lambda_{31} & ? & ? & \lambda_{34} \\
? & ? & \lambda_{43} & \lambda_{44}
\end{array}\right]
$$

where "?" represents the unavailable elements. For notational clarity, for all $r \in \Lambda$, we denote $I_{\kappa}^{r}=\left\{s: \lambda_{r s}\right.$ is known $\}$, $I_{u \kappa}^{r}=\left\{s: \lambda_{r s}\right.$ is unknown $\}$. Moreover, if $I_{\mathcal{\kappa}}^{r} \neq \emptyset$ and have $m$ elements, $I_{\kappa}^{r}$ and $I_{\mathcal{L}}^{r}$ can be further described as $I_{\kappa}^{r}=$ $\left\{\kappa_{1}^{r}, \ldots, \kappa_{m}^{r}\right\}, I_{u \kappa}^{r}=\left\{\bar{\kappa}_{1}^{r}, \ldots, \bar{\kappa}_{M-m}^{r}\right\}$. Where $\kappa_{m}^{r} \in Z^{+}$represents the $m$ th known element with the index $\kappa_{m}^{r}$ in the $r$ th row of matrix $\Pi$ and $\bar{\kappa}_{M-m}^{r} \in Z^{+}$represents the $(M-m)$ th unknown element with the index $\bar{\kappa}_{M-m}^{r}$ in the $r$ th row of matrix $\Pi$.

2.2. Random Packet Dropout. The Bernoulli distributed white sequence $\alpha_{k}$ is introduced to reflect the random packet dropout from the sensor to the controller, which takes value in $\{0,1\}$ and satisfies

$$
\begin{gathered}
\operatorname{Prob}\left\{\alpha_{k}=1\right\}=E\left\{\alpha_{k}\right\}=\bar{\alpha}, \quad 0 \leq \bar{\alpha} \leq 1, \\
\operatorname{Prob}\left\{\alpha_{k}=0\right\}=1-E\left\{\alpha_{k}\right\}=1-\bar{\alpha}, \\
\operatorname{var}\left\{\alpha_{k}\right\}=E\left\{\left(\alpha_{k}-\bar{\alpha}\right)^{2}\right\}=(1-\bar{\alpha}) \bar{\alpha}=\alpha_{1} .
\end{gathered}
$$

Assumption 3. The random variables $\alpha_{k}$ and $d_{k}$ are independent of each other.

In this paper, the measurement with packet dropout and random delay is described by

$$
\bar{y}(k)=\left(1-\alpha_{k}\right) y\left(k-d_{k}\right)+\alpha_{k} \bar{y}(k-1) .
$$

By $\alpha_{k}=0$, we mean that the delayed packet is correctly delivered; that is, $\bar{y}(k)=y\left(k-d_{k}\right)$. While by $\alpha_{k}=1$, we mean that the packet is lost; that is $\bar{y}(k)=\bar{y}(k-1)$.

Remark 4. If the random delay is transformed into the timevarying delay, the model (6) is the same as the one used in [15]. If the transmission delay $d_{k}$ is not taken into account, then (6) reduces to the one used in $[6,7]$. This, in turn, implies that the model $(6)$ is more general than those in $[6,7,15]$. 
The output feedback controller to be designed is of the following form:

$$
\begin{gathered}
x_{c}(k+1)=A x_{c}(k)+B u(k)+L\left(\bar{y}(k)-C x_{c}(k)\right) \\
u(k)=K x_{c}(k),
\end{gathered}
$$

where the gain matrices $L$ and $K$ will be designed later.

Define that

$$
e(k)=x(k)-x_{c}(k) .
$$

Substituting (6), (7) into (8), we have

$$
\begin{aligned}
e(k+1)= & {[(A-L C) e(k)+L C x(k)} \\
& \left.-(1-\bar{\alpha}) L C x\left(k-d_{k}\right)-\bar{\alpha} L \bar{y}(k-1)\right] \\
& +\left(\alpha_{k}-\bar{\alpha}\right)\left[L C x\left(k-d_{k}\right)-L \bar{y}(k-1)\right] \\
& +f(x(k)) .
\end{aligned}
$$

Seting $\xi(k)=\left[\begin{array}{lll}x^{T}(k) & e^{T}(k) & \bar{y}^{T}(k-1)\end{array}\right]^{T}$, we obtain the augmented NCSs with random packet dropout and random delay:

$$
\xi(k+1)=\bar{A} \xi(k)+\bar{B} \xi\left(k-d_{k}\right)+\bar{C} f(x(k)),
$$

where

$$
\begin{gathered}
\bar{A}=A_{1}+\left(\alpha_{k}-\bar{\alpha}\right) A_{2}, \\
\bar{B}=\left[(1-\bar{\alpha})+\left(\bar{\alpha}-\alpha_{k}\right)\right] B_{1}, \\
A_{1}=\left[\begin{array}{ccc}
A+B K & -B K & 0 \\
L C & A-L C & -\bar{\alpha} L \\
0 & 0 & \bar{\alpha}
\end{array}\right], \\
A_{2}=\left[\begin{array}{lll}
0 & 0 & 0 \\
0 & 0 & -L \\
0 & 0 & -I
\end{array}\right], \\
B_{1}=\left[\begin{array}{ccc}
0 & 0 & 0 \\
-L C & 0 & 0 \\
C & 0 & 0
\end{array}\right], \quad \bar{C}=\left[\begin{array}{l}
I \\
I \\
0
\end{array}\right] .
\end{gathered}
$$

\section{Main Results}

In this section, we will present our main results. To this end, the following definition and lemmas will be introduced.

Definition 5. The closed-loop system (10) is said to be stochastically stable if for any initial condition $x_{0} \in R^{n}$ and $d_{0} \in \Lambda$, the following inequality

$$
E\left\{\sum_{k=0}^{\infty}\|x(k)\|^{2} \mid x_{0}, d_{0}\right\}<\infty \text { holds. }
$$

Lemma 6 (see [16]). For given scalar $\lambda_{i} \geq 0$ and matrix $P_{i} \geq 0$, $i=1,2, \ldots, N$, one has

$$
\sum_{i=1}^{N} \lambda_{i} P_{i} \leq \sum_{i=1}^{N} \lambda_{i} \sum_{i=1}^{N} P_{i}
$$

Lemma 7 (see [17]). For any positive definite matrix $W \in$ $R^{n \times n}$, two positive integers $r$ and $r_{0}$ satisfying $r \geq r_{0} \geq 1$, and vector function $x(i) \in R^{n}$, one has

$$
\left(\sum_{i=r_{0}}^{r} x(i)\right)^{T} W\left(\sum_{i=r_{0}}^{r} x(i)\right) \leq \widetilde{r} \sum_{i=r_{0}}^{r} x^{T}(i) W x(i),
$$

where $\tilde{r}=r-r_{0}+1$

In the following theorem, we will derive a sufficient condition such that the closed-loop system (10) is stochastically stable.

Theorem 8. For the given scalars $0 \leq \bar{\alpha} \leq 1, \beta>0$, and matrices $K, L$, the closed-loop system $(10)$ is stochastically stable for each mode $r \in \Lambda$, if there exist matrices $P_{1}(r)>0, P_{2}(r)>$ $0, P_{3}(r)>0, Z_{1}>0, Z_{2}>0, Q_{1}>0, Q_{2}>0, Q_{3}>0$ and scalar $\Gamma>0$ satisfying the following linear matrix inequality:

$$
\left[\begin{array}{ccccccc}
\Phi_{11} & \Phi_{12} & \Phi_{13} & \Phi_{14} & Z_{2} & 0 & \Phi_{17} \\
* & \Phi_{22} & \Phi_{23} & \Phi_{24} & 0 & 0 & \Phi_{27} \\
* & * & \Phi_{33} & 0 & 0 & 0 & \Phi_{37} \\
* & * & * & \Phi_{44} & \Phi_{45} & \Phi_{46} & \Phi_{47} \\
* & * & * & * & \Phi_{55} & 0 & 0 \\
* & * & * & * & * & \Phi_{66} & 0 \\
* & * & * & * & * & * & \Phi_{77}
\end{array}\right]<0,
$$

where

$$
\begin{aligned}
\Phi_{11}= & (A+B K)^{T} \bar{P}_{1}(s)(A+B K)-P_{1}(r) \\
& +(L C)^{T} \bar{P}_{2}(s)(L C)-Z_{2} \\
& +\left(d_{M}-d_{m}+1\right) Q_{1}+Q_{2}+Q_{3} \\
& +\left(d_{M}-d_{m}\right)^{2}(A+B K-I)^{T} Z_{1}(A+B K-I) \\
& +d_{m}^{2}(A+B K-I)^{T} Z_{2}(A+B K-I) \\
& +\Gamma \beta^{2} G^{T} G, \\
\Phi_{12}= & -(A+B K)^{T} \bar{P}_{1}(s)(B K) \\
& +(L C)^{T} \bar{P}_{2}(s)(A-L C) \\
& -d_{m}^{2}(A+B K-I)^{T} Z_{2}(B K) \\
& -\left(d_{M}-d_{m}\right)^{2}(A+B K-I)^{T} Z_{1}(B K), \\
\Phi_{13}= & -\bar{\alpha}(L C)^{T} \bar{P}_{2}(s) L, \\
\Phi_{14}= & -(1-\bar{\alpha})(L C)^{T} \bar{P}_{2}(s)(L C),
\end{aligned}
$$




$$
\begin{aligned}
& \Phi_{17}=(A+B K)^{T} \bar{P}_{1}(s)+(L C)^{T} \bar{P}_{2}(s) \\
& +\left(d_{M}-d_{m}\right)^{2}(A+B K-I)^{T} Z_{1} \\
& +d_{m}^{2}(A+B K-I)^{T} Z_{2}, \\
& \Phi_{22}=(B K)^{T} \bar{P}_{1}(s)(B K) \\
& +(A-L C)^{T} \bar{P}_{2}(s)(A-L C) \\
& +\left(d_{M}-d_{m}\right)^{2}(B K)^{T} Z_{1}(B K) \\
& +d_{m}^{2}(B K)^{T} Z_{2}(B K)-P_{2}(r), \\
& \Phi_{23}=-\bar{\alpha}(A-L C)^{T} \bar{P}_{2}(s) L, \\
& \Phi_{24}=-(1-\bar{\alpha})(A-L C)^{T} \bar{P}_{2}(s)(L C), \\
& \Phi_{27}=-(B K)^{T} \bar{P}_{1}(s)+(A-L C)^{T} \bar{P}_{2}(s) \\
& -\left(d_{M}-d_{m}\right)^{2}(B K)^{T} Z_{1}-d_{m}^{2}(B K)^{T} Z_{2}, \\
& \Phi_{33}=\bar{\alpha}^{2} L^{T} \bar{P}_{2}(s) L+\alpha_{1} L^{T} \bar{P}_{2}(s) L \\
& +\bar{\alpha}^{2} \bar{P}_{3}(s)+\alpha_{1} \bar{P}_{3}(s)-P_{3}(r), \\
& \Phi_{37}=-\bar{\alpha} L^{T} \bar{P}_{2}(s), \\
& \Phi_{44}=(1-\bar{\alpha})^{2}(L C)^{T} \bar{P}_{2}(s)(L C) \\
& +\alpha_{1}(L C)^{T} \bar{P}_{2}(s)(L C)+(1-\bar{\alpha})^{2} C^{T} \bar{P}_{3}(s) C \\
& +\alpha_{1} C^{T} \bar{P}_{3}(s) C-Q_{1}-2 Z_{1}, \\
& \Phi_{45}=Z_{1}, \\
& \Phi_{46}=Z_{1} \text {, } \\
& \Phi_{47}=-(1-\bar{\alpha})(L C)^{T} \bar{P}_{2}(s), \\
& \Phi_{55}=-Q_{2}-Z_{1}-Z_{2}, \\
& \Phi_{66}=-Q_{3}-Z_{1} \text {, } \\
& \Phi_{77}=\bar{P}_{1}(s)+\bar{P}_{2}(s)+\left(d_{M}-d_{m}\right)^{2} Z_{1} \\
& +d_{m}^{2} Z_{2}-\Gamma I \text {. }
\end{aligned}
$$

Proof. Choose the Lyapunov function candidate as

$$
\begin{gathered}
V\left(x(k), d_{k}, \alpha_{k}\right)=\sum_{i=1}^{5} V_{i}\left(x(k), d_{k}, \alpha_{k}\right), \\
V_{1}\left(x(k), d_{k}, \alpha_{k}\right)=x^{T}(k) P_{1}\left(d_{k}\right) x(k) \\
+e^{T}(k) P_{2}\left(d_{k}\right) e(k) \\
+\bar{y}^{T}(k-1) P_{3}\left(d_{k}\right) \bar{y}(k-1),
\end{gathered}
$$

$$
\begin{gathered}
V_{2}\left(x(k), d_{k}, \alpha_{k}\right)=\sum_{i=k-d_{k}}^{k-1} x^{T}(i) Q_{1} x(i) \\
V_{3}\left(x(k), d_{k}, \alpha_{k}\right)=\sum_{i=k-d_{m}}^{k-1} x^{T}(i) Q_{2} x(i) \\
+\sum_{i=k-d_{M}}^{k-1} x^{T}(i) Q_{3} x(i), \\
V_{4}\left(x(k), d_{k}, \alpha_{k}\right)=\sum_{j=-d_{M}+1}^{-d_{m}} \sum_{i=k+j}^{k-1} x^{T}(i) Q_{1} x(i) \\
V_{5}\left(x(k), d_{k}, \alpha_{k}\right) \\
=\sum_{j=-d_{M}+1 i=k+j-1}^{-d_{m}} \sum_{j=-d_{m}}^{k-1}\left(d_{M}-d_{m}\right) \eta^{T}(i) Z_{1} \eta(i) \\
+\sum_{j+j}^{-1} d_{m} \eta^{T}(i) Z_{2} \eta(i),
\end{gathered}
$$

where $\eta(k)=x(k+1)-x(k)=(A+B K-I) x(k)-B K e(k)+$ $f(x(k))$.

Then, for $d_{k+1}=s, d_{k}=r, \bar{P}(s)=\sum_{s=d_{m}}^{d_{M}} \lambda_{r s} P(s)$, we have

$$
\begin{aligned}
& E\left\{\Delta V_{1}\right\}=[(A+B K) x(k)-B K e(k)+f(x(k))]^{T} \\
& \times \bar{P}_{1}(s)[(A+B K) x(k)-B K e(k)+f(x(k))] \\
& -x^{T}(k) P_{1}(r) x(k) \\
& +[(A-L C) e(k)+L C x(k) \\
& -(1-\bar{\alpha}) L C x(k-r)-\bar{\alpha} L \bar{y}(k-1)]^{T} \\
& \times \bar{P}_{2}(s)[(A-L C) e(k)+L C x(k) \\
& -(1-\bar{\alpha}) L C x(k-r)-\bar{\alpha} L \bar{y}(k-1)] \\
& +2[(A-L C) e(k)+L C x(k) \\
& -(1-\bar{\alpha}) L C x(k-r)-\bar{\alpha} L \bar{y}(k-1)]^{T} \\
& \times \bar{P}_{2}(s) f(x(k)) \\
& +\alpha_{1}[L C x(k-r)-L \bar{y}(k-1)]^{T} \\
& \times \bar{P}_{2}(s)[L C x(k-r)-L \bar{y}(k-1)] \\
& +f^{T}(x(k)) \bar{P}_{2}(s) f(x(k)) \\
& -e^{T}(k) P_{2}(r) e(k) \\
& -\bar{y}^{T}(k-1) P_{3}(r) \bar{y}(k-1) \\
& +[(1-\bar{\alpha}) C x(k-r)+\bar{\alpha} \bar{y}(k-1)]^{T} \\
& \times \bar{P}_{3}(s)[(1-\bar{\alpha}) C x(k-r)+\bar{\alpha} \bar{y}(k-1)]
\end{aligned}
$$




$$
\begin{aligned}
& +\alpha_{1}[\bar{y}(k-1)-C x(k-r)]^{T} \\
& \times \bar{P}_{3}(s)[\bar{y}(k-1)-C x(k-r)],
\end{aligned}
$$

$$
\begin{aligned}
E\left\{\Delta V_{2}\right\}= & x^{T}(k) Q_{1} x(k)-x^{T}(k-r) Q_{1} x(k-r) \\
& +\sum_{i=k+1-d_{k+1}}^{k-1} x^{T}(i) Q_{1} x(i) \\
& -\sum_{i=k+1-d_{k}}^{k-1} x^{T}(i) Q_{1} x(i) .
\end{aligned}
$$

Note that

$$
\begin{aligned}
\sum_{i=k+1-d_{k+1}}^{k-1} x^{T}(i) Q_{1} x(i)= & \sum_{i=k+1-d_{m}}^{k-1} x^{T}(i) Q_{1} x(i) \\
& +\sum_{i=k+1-d_{k+1}}^{k-d_{m}} x^{T}(i) Q_{1} x(i) \\
\leq & \sum_{i=k+1-d_{k}}^{k-1} x^{T}(i) Q_{1} x(i) \\
& +\sum_{i=k+1-d_{M}}^{k-d_{m}} x^{T}(i) Q_{1} x(i) .
\end{aligned}
$$

Therefore, we have

$$
\begin{aligned}
E\left\{\Delta V_{2}\right\} \leq & x^{T}(k) Q_{1} x(k)-x^{T}(k-r) Q_{1} x(k-r) \\
& -\sum_{i=k+1-d_{M}}^{k-d_{m}} x^{T}(i) Q_{1} x(i) \\
E\left\{\Delta V_{3}\right\}= & x^{T}(k) Q_{2} x(k)-x^{T}\left(k-d_{m}\right) Q_{2} x\left(k-d_{m}\right) \\
& +x^{T}(k) Q_{3} x(k)-x^{T}\left(k-d_{M}\right) Q_{3} x\left(k-d_{M}\right)
\end{aligned}
$$

$$
\begin{aligned}
E\left\{\Delta V_{4}\right\}= & \left(d_{M}-d_{m}\right) x^{T}(k) Q_{1} x(k) \\
& -\sum_{i=k+1-d_{M}}^{k-d_{m}} x^{T}(i) Q_{1} x(i) \\
E\left\{\Delta V_{5}\right\}= & \left(d_{M}-d_{m}\right)^{2} \eta^{T}(k) Z_{1} \eta(k) \\
& -\sum_{i=k-d_{M}}^{k-d_{m}-1}\left(d_{M}-d_{m}\right) \eta^{T}(i) Z_{1} \eta(i) \\
& +d_{m}^{2} \eta^{T}(k) Z_{2} \eta(k) \\
& -\sum_{i=k-d_{m}}^{k-1} d_{m} \eta^{T}(i) Z_{2} \eta(i) .
\end{aligned}
$$

Using Lemma 7, we have the following inequality

$$
\begin{aligned}
-\sum_{i=k-d_{m}}^{k-1} d_{m} \eta^{T}(i) Z_{2} \eta(i)-\sum_{i=k-d_{M}}^{k-d_{m}-1}\left(d_{M}-d_{m}\right) \eta^{T}(i) Z_{1} \eta(i) \\
=-\sum_{i=k-d_{m}}^{k-1} d_{m} \eta^{T}(i) Z_{2} \eta(i) \\
-\sum_{i=k-r}^{k-d_{m}-1}\left(d_{M}-d_{m}\right) \eta^{T}(i) Z_{1} \eta(i) \\
-\sum_{i=k-d_{M}}^{k-r-1}\left(d_{M}-d_{m}\right) \eta^{T}(i) Z_{1} \eta(i) \\
\leq-\left[x(k)-x\left(k-d_{m}\right)\right]^{T} \\
\quad \times Z_{2}\left[x(k)-x\left(k-d_{m}\right)\right] \\
-\left[x\left(k-d_{m}\right)-x(k-r)\right]^{T} \\
\quad \times Z_{1}\left[x\left(k-d_{m}\right)-x(k-r)\right] \\
-\left[x(k-r)-x\left(k-d_{M}\right)\right]^{T} \\
\times Z_{1}\left[x(k-r)-x\left(k-d_{M}\right)\right]
\end{aligned}
$$

From (2), for any scalar $\Gamma>0$, we have

$$
\Gamma\left(\beta^{2} x^{T}(k) G^{T} G x(k)-f^{T}(x(k)) f(x(k))\right) \geq 0 .
$$

Then, from (18), (21)-(26), we have

$$
E\left\{\Delta V\left(x(k), d_{k}, \alpha_{k}\right)\right\} \leq \zeta^{T} \Phi \zeta,
$$

where

$\zeta(k)$

$$
=\left[\begin{array}{lllll}
\xi^{T}(k) & x^{T}(k-r) & x^{T}\left(k-d_{m}\right) & x^{T}\left(k-d_{M}\right) & f^{T}(x(k))
\end{array}\right]^{T}
$$

$$
\Phi=\left[\begin{array}{ccccccc}
\Phi_{11} & \Phi_{12} & \Phi_{13} & \Phi_{14} & Z_{2} & 0 & \Phi_{17} \\
* & \Phi_{22} & \Phi_{23} & \Phi_{24} & 0 & 0 & \Phi_{27} \\
* & * & \Phi_{33} & 0 & 0 & 0 & \Phi_{37} \\
* & * & * & \Phi_{44} & \Phi_{45} & \Phi_{46} & \Phi_{47} \\
* & * & * & * & \Phi_{55} & 0 & 0 \\
* & * & * & * & * & \Phi_{66} & 0 \\
* & * & * & * & * & * & \Phi_{77}
\end{array}\right]
$$

From (15), we conclude that $\Phi<0$; thus we have

$$
E\left\{\Delta V\left(x(k), d_{k}, \alpha_{k}\right)\right\} \leq \zeta^{T} \Phi \zeta<0 .
$$

Following a similar line in the proof of Theorem 1 in [18], the stochastic stability of the closed-loop system (10) can be confirmed.

Now, we apply the result obtained in Theorem 8 to solve the controller design problem. 
Theorem 9. For the given scalars $0 \leq \bar{\alpha} \leq 1, \beta>0, \varepsilon_{l}>$ $0, l=1,2,3,4,5$, the closed-loop system (10) is stochastically stable for each mode $r \in \Lambda$, if there exist matrices $X_{1}(r)>0$,
$X_{2}(r)>0, X_{3}(r)>0, Z_{1}>0, Z_{2}>0, Q_{1}>0, Q_{2}>0$, $Q_{3}>0, K, L$ and scalar $\Gamma>0$, satisfying the following linear matrix inequality:

$$
\left[\begin{array}{ccccccccccccc}
\bar{\Omega}_{0} & \Omega_{1} & \Omega_{2} & \Omega_{3} & \bar{\Omega}_{3} & \Omega_{4} & \bar{\Omega}_{4} & \Omega_{5} & \bar{\Omega}_{5} & \Omega_{6} & \bar{\Omega}_{6} & \Omega_{7} & \bar{\Omega}_{7} \\
* & -2 \varepsilon_{1} I+\varepsilon_{1}^{2} Z_{1} & 0 & 0 & 0 & 0 & 0 & 0 & 0 & 0 & 0 & 0 & 0 \\
* & * & -2 \varepsilon_{2} I+\varepsilon_{2}^{2} Z_{2} & 0 & 0 & 0 & 0 & 0 & 0 & 0 & 0 & 0 & 0 \\
* & * & * & \Omega_{8} & 0 & 0 & 0 & 0 & 0 & 0 & 0 & 0 & 0 \\
* & * & * & * & \bar{\Omega}_{8} & 0 & 0 & 0 & 0 & 0 & 0 & 0 & 0 \\
* & * & * & * & * & \Omega_{9} & 0 & 0 & 0 & 0 & 0 & 0 & 0 \\
* & * & * & * & * & * & \bar{\Omega}_{9} & 0 & 0 & 0 & 0 & 0 & 0 \\
* & * & * & * & * & * & * & \Omega_{9} & 0 & 0 & 0 & 0 & 0 \\
* & * & * & * & * & * & * & * & \bar{\Omega}_{9} & 0 & 0 & 0 & 0 \\
* & * & * & * & * & * & * & * & * & \Omega_{10} & 0 & 0 & 0 \\
* & * & * & * & * & * & * & * & * & * & \bar{\Omega}_{10} & 0 & 0 \\
* & * & * & * & * & * & * & * & * & * & * & \Omega_{10} & 0 \\
* & * & * & * & * & * & * & * & * & * & * & * & \bar{\Omega}_{10}
\end{array}\right],
$$

where

$$
\bar{\Omega}_{0}=\left[\begin{array}{ccccccc}
\overline{\bar{\Phi}}_{11} & 0 & 0 & 0 & Z_{2} & 0 & 0 \\
* & -2 \varepsilon_{4} I+\varepsilon_{4}^{2} X_{2}(r) & 0 & 0 & 0 & 0 & 0 \\
* & * & -2 \varepsilon_{5} I+\varepsilon_{5}^{2} X_{3}(r) & 0 & 0 & 0 & 0 \\
* & * & * & -Q_{1}-2 Z_{1} & \Phi_{45} & \Phi_{46} & 0 \\
* & * & * & * & \Phi_{55} & 0 & 0 \\
* & * & * & * & * & \Phi_{66} & 0 \\
* & * & * & * & * & * & -\Gamma I
\end{array}\right],
$$

$$
\begin{aligned}
& \overline{\bar{\Phi}}_{11}=-2 \varepsilon_{3} I+\varepsilon_{3}^{2} X_{1}(r)-Z_{2}+\left(d_{M}-d_{m}+1\right) Q_{1}+Q_{2}+Q_{3}+\Gamma \beta^{2} G^{T} G, \quad P^{-1}(r)=X(r), \\
& \Theta_{1}=\left[\begin{array}{lll}
\sqrt{\lambda_{r \kappa_{1}^{r}}} \cdots & \sqrt{\lambda_{r \kappa_{m}^{r}}}
\end{array}\right], \quad \Theta_{2}=\left[\begin{array}{lll}
\sqrt{v_{r}} & \cdots & \sqrt{v_{r}}
\end{array}\right],
\end{aligned}
$$

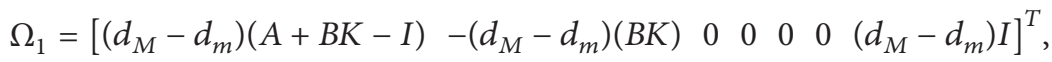

$$
\begin{aligned}
& \Omega_{2}=\left[\begin{array}{llllllll}
d_{m}(A+B K-I) & -d_{m}(B K) & 0 & 0 & 0 & 0 & \left.d_{m} I\right]^{T},
\end{array}\right. \\
& \Omega_{3}=\Theta_{1}[(A+B K)-B K \quad 0 \quad 00 \quad 0 \quad 001]^{T},
\end{aligned}
$$

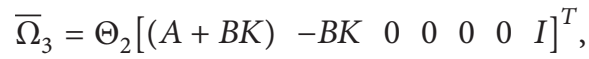

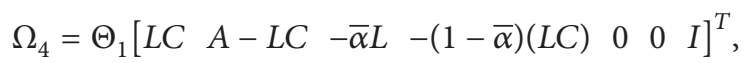

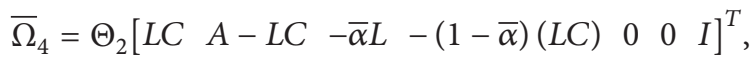

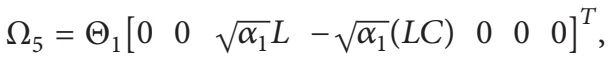

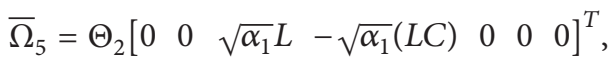

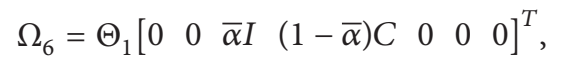

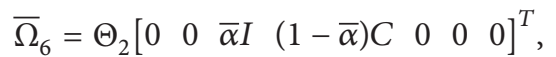




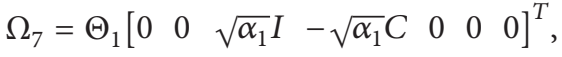

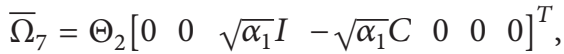

$$
\begin{aligned}
& \Omega_{8}=-\operatorname{diag}\left\{X_{1}\left(\kappa_{1}^{r}\right) \cdots X_{1}\left(\kappa_{m}^{r}\right)\right\}, \quad \bar{\Omega}_{8}=-\operatorname{diag}\left\{X_{1}\left(\bar{\kappa}_{1}^{r}\right) \cdots X_{1}\left(\bar{\kappa}_{M-m}^{r}\right)\right\}, \\
& \Omega_{9}=-\operatorname{diag}\left\{X_{2}\left(\kappa_{1}^{r}\right) \cdots X_{2}\left(\kappa_{m}^{r}\right)\right\}, \quad \bar{\Omega}_{9}=-\operatorname{diag}\left\{X_{2}\left(\bar{\kappa}_{1}^{r}\right) \cdots X_{2}\left(\bar{\kappa}_{M-m}^{r}\right)\right\}, \\
& \Omega_{10}=-\operatorname{diag}\left\{X_{3}\left(\kappa_{1}^{r}\right) \cdots X_{3}\left(\kappa_{m}^{r}\right)\right\}, \quad \bar{\Omega}_{10}=-\operatorname{diag}\left\{X_{3}\left(\bar{\kappa}_{1}^{r}\right) \cdots X_{3}\left(\bar{\kappa}_{M-m}^{r}\right)\right\} .
\end{aligned}
$$

Proof. Note that (15) is equivalent to

$$
\begin{aligned}
& {\left[\begin{array}{ccccccc}
\Phi_{11} & 0 & 0 & 0 & Z_{2} & 0 & 0 \\
* & -P_{2}(r) & 0 & 0 & 0 & 0 & 0 \\
* & * & -P_{3}(r) & 0 & 0 & 0 & 0 \\
* & * & * & -Q_{1}-2 Z_{1} & \Phi_{45} & \Phi_{46} & 0 \\
* & * & * & * & \Phi_{55} & 0 & 0 \\
* & * & * & * & * & \Phi_{66} & 0 \\
* & * & * & * & * & * & -\Gamma I
\end{array}\right]} \\
& +\left[\begin{array}{lllllll}
A+B K & -B K & 0 & 0 & 0 & 0 & I
\end{array}\right]^{T} \bar{P}_{1}(s) \\
& \times[A+B K-B K \quad 0 \quad 000001]
\end{aligned}
$$

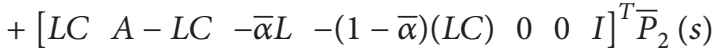

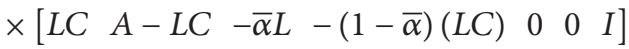

$$
\begin{aligned}
& +\left[\begin{array}{lllllll}
0 & 0 & \sqrt{\alpha_{1}} L & -\sqrt{\alpha_{1}}(L C) & 0 & 0 & 0
\end{array}\right]^{T} \bar{P}_{2}(s) \\
& \times\left[\begin{array}{lllllll}
0 & 0 & \sqrt{\alpha_{1}} L & -\sqrt{\alpha_{1}}(L C) & 0 & 0 & 0
\end{array}\right]
\end{aligned}
$$

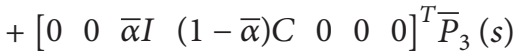

$$
\begin{aligned}
& \times\left[\begin{array}{llll}
0 & 0 & \bar{\alpha} I & (1-\bar{\alpha}) C \\
C & 0 & 0 & 0
\end{array}\right]
\end{aligned}
$$

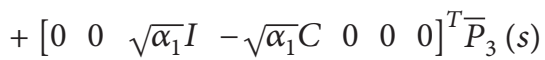

$$
\begin{aligned}
& \times\left[\begin{array}{lllllll}
0 & 0 & \sqrt{\alpha_{1}} I & -\sqrt{\alpha_{1}} C & 0 & 0 & 0
\end{array}\right]
\end{aligned}
$$

$$
\begin{aligned}
& +\left[\begin{array}{llllllll}
d_{m}(A+B K-I) & -d_{m}(B K) & 0 & 0 & 0 & 0 & d_{m} I
\end{array}\right]^{T} Z_{2} \\
& \times\left[\begin{array}{llllllll}
d_{m}(A+B K-I) & -d_{m}(B K) & 0 & 0 & 0 & 0 & d_{m} I
\end{array}\right] \\
& +\left[\begin{array}{c}
\left(d_{M}-d_{m}\right)(A+B K-I)^{T} \\
-\left(d_{M}-d_{m}\right)(B K)^{T} \\
0 \\
0 \\
0 \\
\left(d_{M}-d_{m}\right) I
\end{array}\right] Z_{1}\left[\begin{array}{c}
\left(d_{M}-d_{m}\right)(A+B K-I)^{T} \\
-\left(d_{M}-d_{m}\right)(B K)^{T} \\
0 \\
0 \\
0 \\
0 \\
\left(d_{M}-d_{m}\right) I
\end{array}\right]^{T}<0,
\end{aligned}
$$

where $\bar{\Phi}_{11}=-P_{1}(r)-Z_{2}+\left(d_{M}-d_{m}+1\right) Q_{1}+Q_{2}+Q_{3}+\Gamma \beta^{2} G^{T} G$. Via Lemma 6 , we obtain

$$
\begin{aligned}
\sum_{s=1}^{M} \lambda_{r s} P\left(\tau_{s}\right) & =\sum_{s \in I_{\kappa}^{r}} \lambda_{r s} P\left(\tau_{s}\right)+\sum_{s \in I_{u \kappa}^{r}} \lambda_{r s} P\left(\tau_{s}\right) \\
& \leq P\left(I_{\kappa}^{r}\right)+v_{r} P\left(I_{u \kappa}^{r}\right)
\end{aligned}
$$

where

$$
\begin{gathered}
P\left(I_{\kappa}^{r}\right)=\sum_{s \in I_{\kappa}^{r}} \lambda_{r s} P\left(\tau_{s}\right), \quad P\left(I_{u \kappa}^{r}\right)=\sum_{s \in I_{u \kappa}^{r}} P\left(\tau_{s}\right), \\
v_{r}=1-\sum_{s \in I_{\kappa}^{r}} \lambda_{r s} .
\end{gathered}
$$

From (34) and Schur complement Lemma, (33) can be transformed into the following inequality:

$$
\left[\begin{array}{ccccccccccccc}
\Omega_{0} & \Omega_{1} & \Omega_{2} & \Omega_{3} & \bar{\Omega}_{3} & \Omega_{4} & \bar{\Omega}_{4} & \Omega_{5} & \bar{\Omega}_{5} & \Omega_{6} & \bar{\Omega}_{6} & \Omega_{7} & \bar{\Omega}_{7} \\
* & -Z_{1}^{-1} & 0 & 0 & 0 & 0 & 0 & 0 & 0 & 0 & 0 & 0 & 0 \\
* & * & -Z_{2}^{-1} & 0 & 0 & 0 & 0 & 0 & 0 & 0 & 0 & 0 & 0 \\
* & * & * & \Omega_{8} & 0 & 0 & 0 & 0 & 0 & 0 & 0 & 0 & 0 \\
* & * & * & * & \bar{\Omega}_{8} & 0 & 0 & 0 & 0 & 0 & 0 & 0 & 0 \\
* & * & * & * & * & \Omega_{9} & 0 & 0 & 0 & 0 & 0 & 0 & 0 \\
* & * & * & * & * & * & \bar{\Omega}_{9} & 0 & 0 & 0 & 0 & 0 & 0 \\
* & * & * & * & * & * & * & \Omega_{9} & 0 & 0 & 0 & 0 & 0 \\
* & * & * & * & * & * & * & * & \bar{\Omega}_{9} & 0 & 0 & 0 & 0 \\
* & * & * & * & * & * & * & * & * & \Omega_{10} & 0 & 0 & 0 \\
* & * & * & * & * & * & * & * & * & * & \bar{\Omega}_{10} & 0 & 0 \\
* & * & * & * & * & * & * & * & * & * & * & \Omega_{10} & 0 \\
* & * & * & * & * & * & * & * & * & * & * & * & \bar{\Omega}_{10}
\end{array}\right]<0,
$$


where

$$
\Omega_{0}=\left[\begin{array}{ccccccc}
\Phi_{11} & 0 & 0 & 0 & Z_{2} & 0 & 0 \\
* & -P_{2}(r) & 0 & 0 & 0 & 0 & 0 \\
* & * & -P_{3}(r) & 0 & 0 & 0 & 0 \\
* & * & * & -Q_{1}-2 Z_{1} & \Phi_{45} & \Phi_{46} & 0 \\
* & * & * & * & \Phi_{55} & 0 & 0 \\
* & * & * & * & * & \Phi_{66} & 0 \\
* & * & * & * & * & * & -\Gamma I
\end{array}\right] .
$$
hold:

For scalars $\varepsilon_{l}, l=1,2,3,4,5$, the following inequalities

$$
\begin{gathered}
\left(I-\varepsilon_{1} Z_{1}\right)^{T} Z_{1}^{-1}\left(I-\varepsilon_{1} Z_{1}\right) \geq 0, \\
\left(I-\varepsilon_{2} Z_{2}\right)^{T} Z_{2}^{-1}\left(I-\varepsilon_{2} Z_{2}\right) \geq 0, \\
\left(I-\varepsilon_{3} P_{1}^{-1}(r)\right)^{T} P_{1}(r)\left(I-\varepsilon_{3} P_{1}^{-1}(r)\right) \geq 0, \\
\left(I-\varepsilon_{4} P_{2}^{-1}(r)\right)^{T} P_{2}(r)\left(I-\varepsilon_{4} P_{2}^{-1}(r)\right) \geq 0, \\
\left(I-\varepsilon_{5} P_{3}^{-1}(r)\right)^{T} P_{3}(r)\left(I-\varepsilon_{5} P_{3}^{-1}(r)\right) \geq 0 .
\end{gathered}
$$

Then we have

$$
\begin{gathered}
-Z_{1}^{-1} \leq-2 \varepsilon_{1} I+\varepsilon_{1}^{2} Z_{1}, \\
-Z_{2}^{-1} \leq-2 \varepsilon_{2} I+\varepsilon_{2}^{2} Z_{2}, \\
-P_{1}(r) \leq-2 \varepsilon_{3} I+\varepsilon_{3}^{2} P_{1}^{-1}(r), \\
-P_{2}(r) \leq-2 \varepsilon_{4} I+\varepsilon_{4}^{2} P_{2}^{-1}(r), \\
-P_{3}(r) \leq-2 \varepsilon_{5} I+\varepsilon_{5}^{2} P_{3}^{-1}(r) .
\end{gathered}
$$

According to the inequalities (39), it is obtained that if (31) holds, then (36) holds.

Remark 10. In Theorem 9, the inequality (31) is a linear matrix inequality, which can easily be solved by using the Matlab LMI Toolbox.

Remark 11. Compared with the results in $[9,10]$, partially unknown elements in the transition probability matrix do not require the complete knowledge or even the lower and upper bounds of the transition probabilities in the jump process, which means that it is more powerful and desirable.

Remark 12. The results in this paper derived based on the assumption that the network exists only in the sensor-tocontroller side, but when the network exists both in the sensor-to-controller and controller-to-actuator sides, this needs further study.
TABLE 1: The maximum dropout rate $\bar{\alpha}$ for different $\beta$ and $\Pi$.

\begin{tabular}{lccc}
\hline$\beta$ & 0.1 & 0.2 & 0.3 \\
\hline$\Pi_{1}$ & 0.9999 & 0.9865 & 0.9712 \\
$\Pi_{2}$ & 0.8335 & 0.8309 & 0.8151 \\
$\Pi_{3}$ & 0.2793 & - & - \\
\hline
\end{tabular}

\section{Numerical Example}

The system data of (1) is described as follows:

$$
\begin{gathered}
A=\left[\begin{array}{ll}
0.08 & 0.13 \\
0.41 & 0.19
\end{array}\right], \quad B=\left[\begin{array}{l}
1 \\
0
\end{array}\right], \\
C=\left[\begin{array}{cc}
0.2 & 0 \\
0.6 & -0.1
\end{array}\right] .
\end{gathered}
$$

The random delay is $d_{k} \in\{1,2,3\}$, and its transition probability matrix is given by

$$
\begin{gathered}
\Pi_{1}=\left[\begin{array}{ccc}
0.9 & 0.05 & 0.05 \\
0.5 & 0.4 & 0.1 \\
0.1 & 0.1 & 0.8
\end{array}\right], \quad \Pi_{2}=\left[\begin{array}{ccc}
0.9 & ? & ? \\
? & 0.4 & ? \\
0.1 & 0.1 & 0.8
\end{array}\right], \\
\Pi_{3}=\left[\begin{array}{lll}
? & ? & ? \\
? & ? & ? \\
? & ? & ?
\end{array}\right] .
\end{gathered}
$$

The maximum allowable nonlinear level $\beta$ and the maximum packet dropout rate $\bar{\alpha}$ for three different transition probability matrices are given in Tables 1 and 2, respectively, in which "-_" means that the results are not applicable to the corresponding cases. Thus, from Tables 1 and 2, it is easily seen that the more transition probability matrix we have, the larger maximum dropout rate $\bar{\alpha}$ for different $\beta$ and the larger maximum allowable nonlinear level $\beta$ for different $\bar{\alpha}$ can be obtained for ensuring stability.

Give that

$$
\begin{gathered}
x(-2)=x(-1)=x(0)=\left[\begin{array}{ll}
1 & -0.5
\end{array}\right]^{T}, \\
\bar{y}(-2)=\bar{y}(-1)=\bar{y}(0)=\left[\begin{array}{ll}
0 & 0
\end{array}\right]^{T}, \\
\bar{\alpha}=0.5, \quad \beta=0.3, \quad \varepsilon_{1}=3.5, \\
\varepsilon_{2}=2.8, \quad \varepsilon_{3}=10, \quad \varepsilon_{4}=1.1, \\
\varepsilon_{5}=0.6, \quad G=1 .
\end{gathered}
$$

Assuming that the nonlinear function is $f(x(k))=$ $0.1 \sin (x(k))$, by Theorem 9 , we can obtain the controller gain and observer gain with $\Pi_{2}$ as follows:

$$
\begin{aligned}
K & =\left[\begin{array}{ll}
0.0017 & -0.0137
\end{array}\right], \\
L & =\left[\begin{array}{ll}
0.0028 & 0.0020 \\
0.0148 & 0.0361
\end{array}\right] .
\end{aligned}
$$

Based on the designed controller and observer, the simulation results of the state responses are given in Figure 2. It is obvious that the designed novel dynamic output feedback 
TABLE 2: The maximum allowable nonlinear level $\beta$ for different $\bar{\alpha}$ and $\Pi$.

\begin{tabular}{lccc}
\hline $\bar{\alpha}$ & 0.15 & 0.35 & 0.55 \\
\hline$\Pi_{1}$ & 0.3671 & 0.3652 & 0.3640 \\
$\Pi_{2}$ & 0.3302 & 0.3282 & 0.3258 \\
$\Pi_{3}$ & 0.1608 & - & - \\
\hline
\end{tabular}

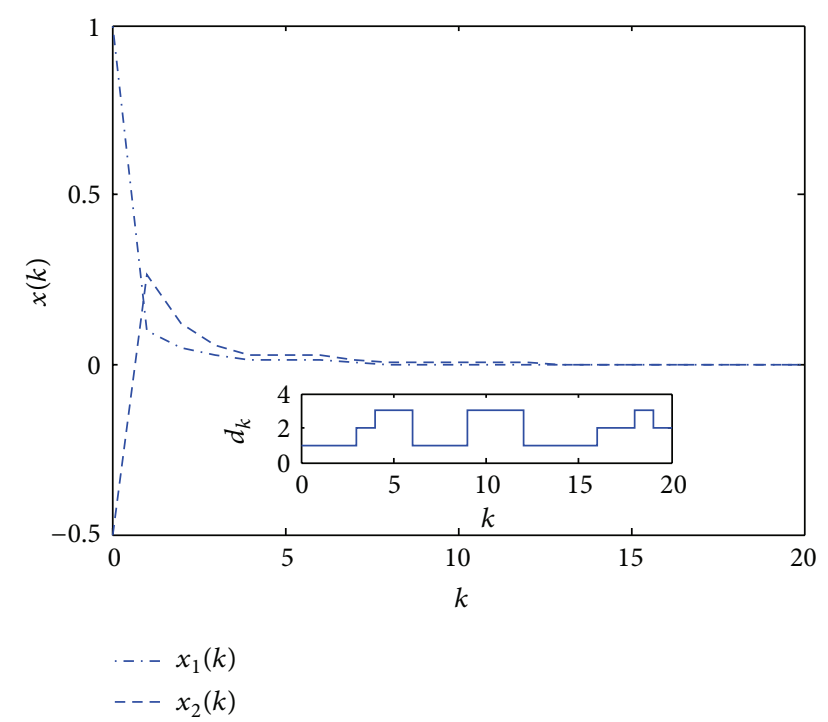

FIgURE 2: State response of the closed-loop system.

controller is feasible and ensures the stability of the closedloop system despite the random packet dropout and random delay.

\section{Conclusion}

In this paper, a novel dynamic output feedback controller is designed for nonlinear networked control systems with both random packet dropout and random delay. A sufficient condition is presented for the stabilization of the closed systems based upon the Lyapunov theory. The quantitative relationship of the maximum dropout rate, the transition probability matrix, and the maximum allowable nonlinear level is derived by solving a set of linear matrix inequalities. An example is presented to illustrate the effectiveness of the proposed method.

\section{Acknowledgments}

This work is supported by NNSFC (60974139), and by the Fundamental Research Funds for the central universities (72103676 and K50511070008).

\section{References}

[1] E. Tian, D. Yue, and X. Zhao, "Quantised control design for networked control systems," IET Control Theory and Applications, vol. 1, no. 6, pp. 1693-1699, 2007.
[2] H. Gao and T. Chen, "A new approach to quantized feedback control systems," Automatica, vol. 44, no. 2, pp. 534-542, 2008.

[3] Y. G. Niu, T. G. Jia, X. Y. Wang, and F. W. Yang, "Outputfeedback control design for NCSs subject to quantization and dropout," Information Sciences, vol. 179, no. 21, pp. 3804-3813, 2009.

[4] M. Y. Fu and L. H. Xie, “The sector bound approach to quantized feedback control," IEEE Transactions on Automatic Control, vol. 50, no. 11, pp. 1698-1711, 2005.

[5] J. G. Li, J. Q. Yuan, and J. G. Lu, "Observer-based $H_{\infty}$ control for networked nonlinear systems with random packet losses," ISA Transactions, vol. 49, no. 1, pp. 39-46, 2010.

[6] W.-A. Zhang, L. Yu, and H. Song, " $H_{\infty}$ filtering of networked discrete-time systems with random packet losses," Information Sciences, vol. 179, no. 22, pp. 3944-3955, 2009.

[7] X. Fang and J. Wang, "Stochastic observer-based guaranteed cost control for networked control systems with packet dropouts," IET Control Theory and Applications, vol. 2, no. 11, pp. 980-989, 2008.

[8] J. L. Xiong and J. Lam, "Stabilization of linear systems over networks with bounded packet loss," Automatica, vol. 43, no. 1, pp. 80-87, 2007.

[9] L. Q. Zhang, Y. Shi, T. W. Chen, and B. Huang, "A new method for stabilization of networked control systems with random delays," IEEE Transactions on Automatic Control, vol. 50, no. 8, pp. 1177-1181, 2005.

[10] Y. Shi and B. Yu, "Output feedback stabilization of networked control systems with random delays modeled by Markov chains," IEEE Transactions on Automatic Control, vol. 54, no. 7, pp. 1668-1674, 2009.

[11] M. Liu, D. W. C. Ho, and Y. Niu, "Stabilization of Markovian jump linear system over networks with random communication delay," Automatica, vol. 45, no. 2, pp. 416-421, 2009.

[12] Y. Halevi and A. Ray, "Integrated communication and control systems. Part 1-Analysis," Journal of Dynamic Systems, Measurement and Control, Transactions of the ASME, vol. 110, no. 4, pp. 367-373, 1988.

[13] G. C. Walsh, O. Beldiman, and L. G. Bushnell, "Asymptotic behavior of nonlinear networked control systems," IEEE Transactions on Automatic Control, vol. 46, no. 7, pp. 1093-1097, 2001.

[14] W. Zhang, M. S. Branicky, and S. M. Phillips, "Stability of networked control systems," IEEE Control Systems Magazine, vol. 21, no. 1, pp. 84-97, 2001.

[15] Y. Zhang and H. Fang, "Stabilization of nonlinear networked systems with sensor random packet dropout and time-varying delay," Applied Mathematical Modelling, vol. 35, no. 5, pp. 22532264, 2011.

[16] G. L. Wang, Q. L. Zhang, and V. Sreeram, "Partially modedependent $H_{\infty}$ filtering for discrete-time Markovian jump systems with partly unknown transition probabilities," Signal Processing, vol. 90, no. 2, pp. 548-556, 2010.

[17] X. F. Jiang, Q.-L. Han, and X. H. Yu, "Stability criteria for linear discrete-time systems with interval-like time-varying delay," in Proceedings of the American Control Conference (ACC '05), pp. 2817-2822, June 2005.

[18] E. K. Boukas and Z. K. Liu, "Robust $H_{\infty}$ control of discrete-time Markovian jump linear systems with mode-dependent timedelays," IEEE Transactions on Automatic Control, vol. 46, no. 12, pp. 1918-1924, 2001. 


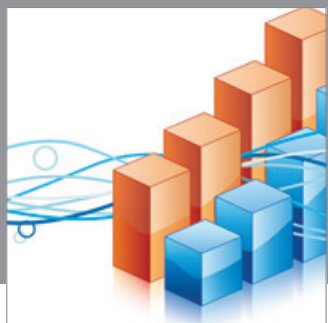

Advances in

Operations Research

mansans

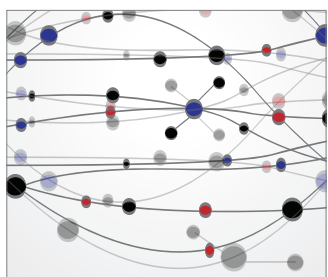

The Scientific World Journal
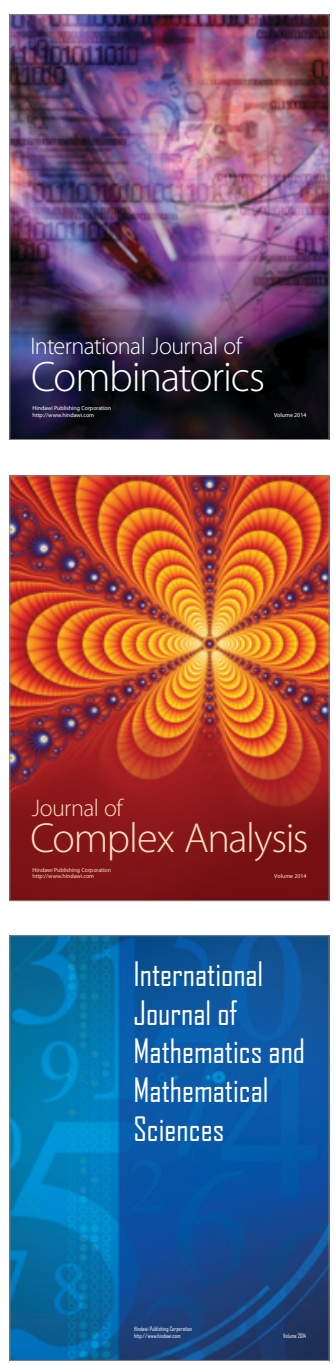
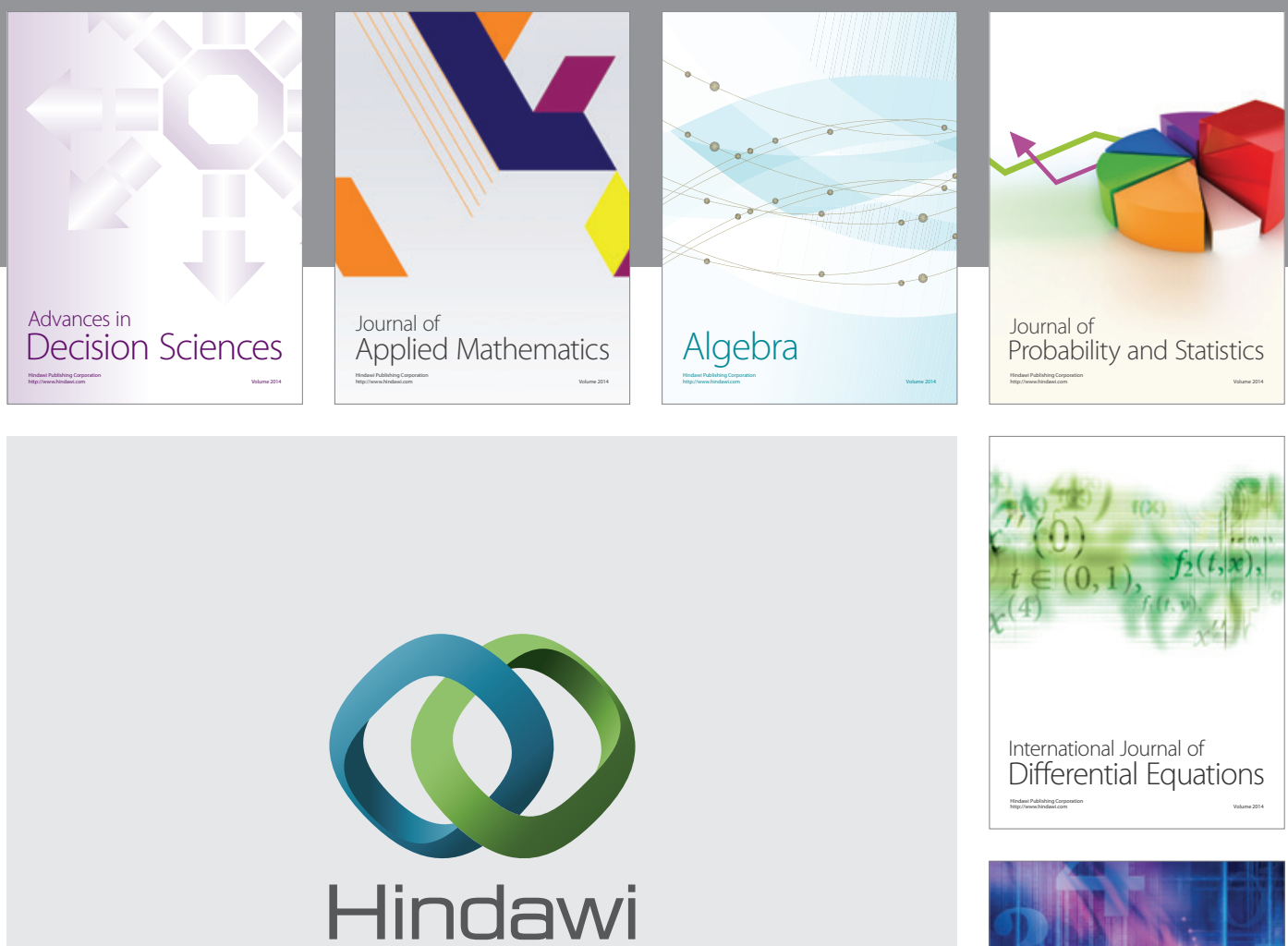

Submit your manuscripts at http://www.hindawi.com
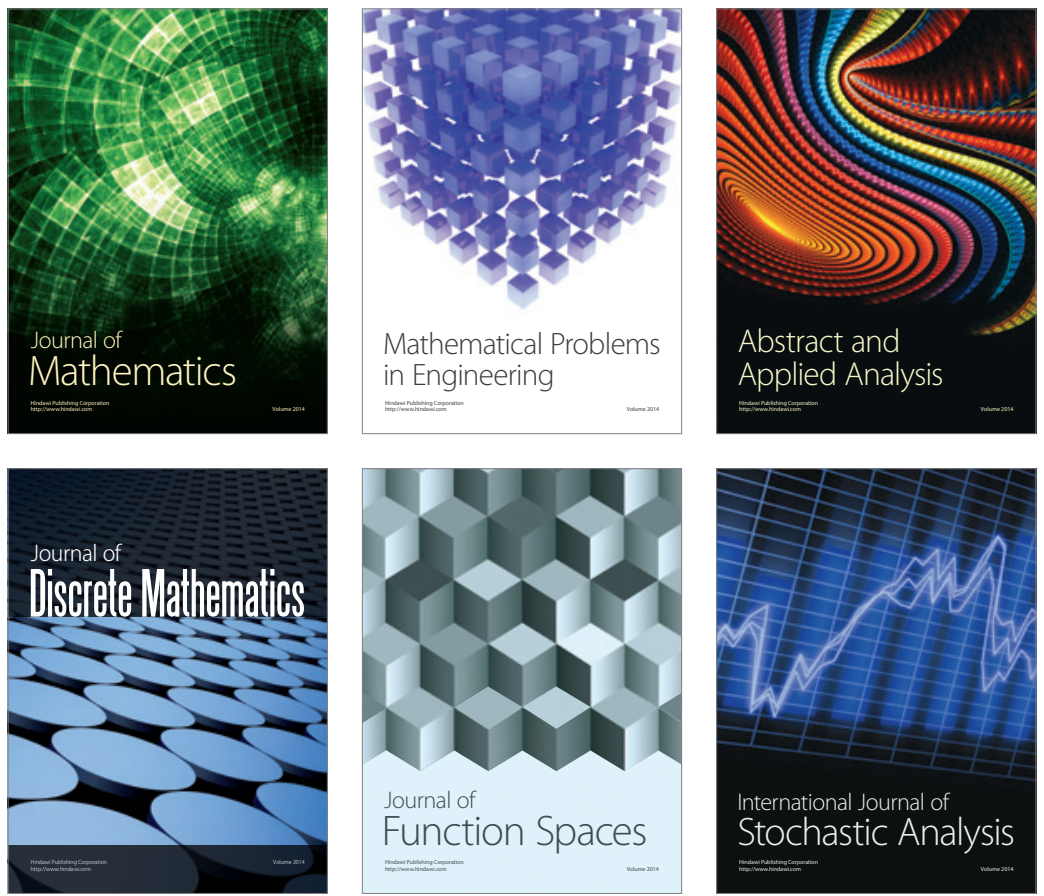

Journal of

Function Spaces

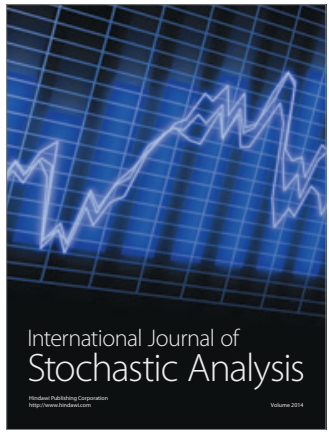

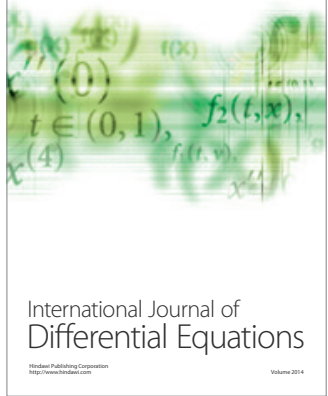
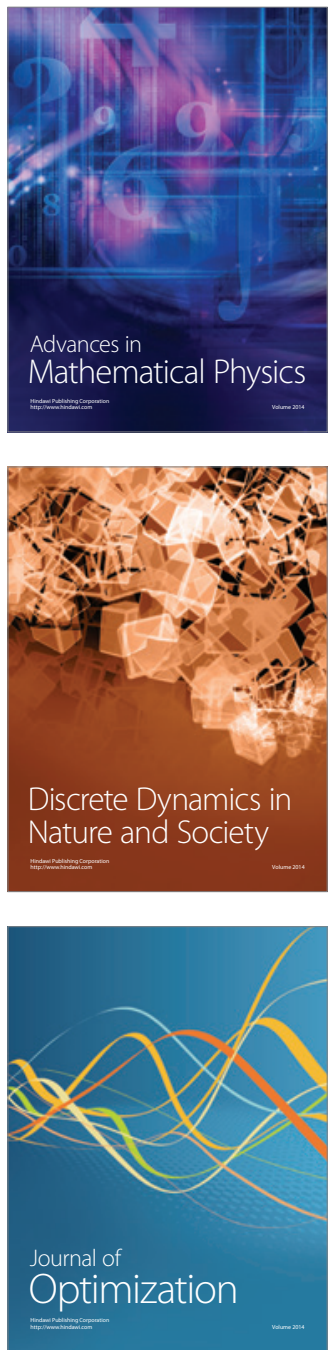